\title{
Articulated Structures with Tendon Actuation for Whole-Limb Manipulation.
}

Paolo Petreschi

\author{
Domenico Prattichizro
}

Centro "E. Piaggio"

Università di Pisa
Antonio Bicchi

\begin{abstract}
We present a general framework for the modelling of a class of mechanical systems for robotic manipulation, consisting of articulated limbs with redundant tendinous actuation and unilateral constraints. Such systems, that include biomorphically designed devices, are regarded as a collection of rigid bodies, interacting through connections that model both joints and contacts with virtual springs. Methods previously developed for the analysis of force distribution in multiple whole-limb manipulation are generalised to this broader class of mechanisms, and are shown to provide a basis for the control of co-contraction and internal forces that guarantee proper operation of the system.
\end{abstract}

\section{Introduction}

The articular and tendinous structure of animal limbs provide an outstanding example of mechanical systems with extremely high performance, that attracts the interest of researchers in physiology, biomaterials, and robotics. The extremely low friction in articular joints and the remotisation of actuators made possible by tendon structures are two of the prominent advantages of biomorphic structures over conventional mechanical design. Besides the exceptional lubrication properties of synovial fluid, low friction in articular joints is achieved through the use of rolling pairs between bone processes. The large redundancy of the tendinous system allows actuators (muscles) to be located far away from articular joints, and offers the possibility of "co-contracting" the limbs so as to optimally tune their stiffness and configure the limbs for different tasks (precision movements, force exertion, etc.).

The analysis of both the kinematics of articular joints and the redundancy in tendinous actuation offer non-trivial difficulties. Among these are the nonholonomy of rolling pairs, and the unilateral action of tendons (no "pushing" is allowed). The literature on tendon-actuated mechanisms is relatively rich in robotics, where they have been used especially in the design of dextrous robotic hands. The necessity to avoid tendon cables to go slack has been often solved most simply by using two tendons per joint in pushpull (or agonistic-antagonistic) configuration ([Jacobsen et al., 1989]). The use of $2 n$ cables and actuators for $n$ joints however hampers the practicality of the design and affects its cost. Morecky et al. [1980] and
Salisbury [1982] described the use of co-contraction in mechanisms using $n+1$ tendons. A general analysis of tendon driven mechanisms has been attempted in most cases for systems where tendon are routed through joints by means of pulleys (see e.g. [Lee and Tsai, 1991]). A qualitative description of more general systems composed of nets of tendons and actuators has been presented by Barbieri and Bergamasco [1991]. More complex models of tendon-actuated systems have been considered in the biomechanical literature ([Mussa Ivaldi et al., 1988]; [Buchner et al., 1988]), and some authors used anthropomorphic models to attack the design of robot hands ([Backer et al., 1986]). Recently, Deno et al. [1992] approached the dynamic modelization and analysis of "finger-like" mechanisms by using graph theory.

The approach proposed in this paper is meant to encompass a wide variety of configurations that can be encountered in biological systems or conceived for artificial devices. For the sake of the widest generality, we model articulated limbs with tendinous actuation and manipulated objects as a collection of rigid bodies, interacting through contacts with characteristic kinematic and visco-elastic properties ([Wittemburg, 1977]). Distinction between manipulator "link" and "objects" to be manipulated is not intrinsic to the model, but can be recovered in the final stage of analysis. Contacts of any of the bodies with others are allowed, so that whole-limb manipulation is naturally investigated in this framework. Also, tinematic rolling pairs are allowed not only between a link and the manipulated object, but also between links in the same limb chain, so as to permit the above mentioned, high-efficiency biomorphic joints to be included in the analysis.

A quasi-static, small-displacement analysis of force distribution between contacts and through tendons for these systems is proposed that follows the lines of [Bicchi, 1993]. As a result of the proposed analysis, one is able to describe how external and internal forces are distributed in the system in a given configuration. Internal forces are defined (as usual in grasp literature) as the set of tendon tensions and contact forces that are self-balanced, i.e., do not affect motions of any part of the system. Among internal forces, "cocontraction" forces are further distinguished as those combinations of tendon tensions that influence contact 


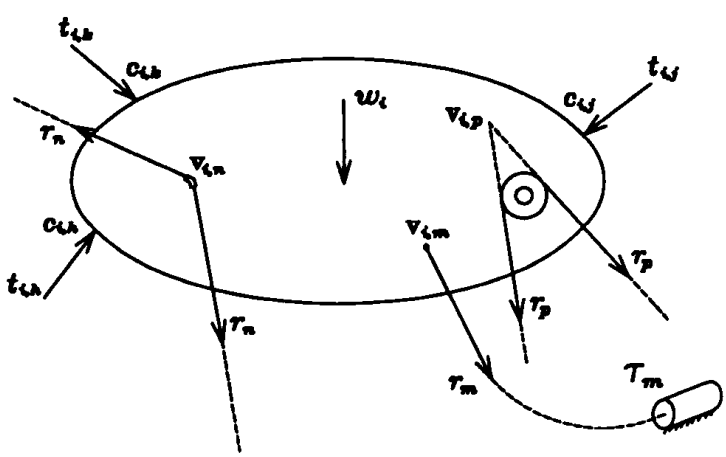

Figure 1: Characteristic connection points, tendon conduits, and forces applied on the $i$-th body.

forces between finger links, but do not act upon manipulated objects. The actual capability of the system to actively control such forces is investigated. By these means, a basis for the choice of internal forces that allow to avoid loosing the grip on manipulated objects as well as a basis for co-contraction forces to avoid tendon backlash and joint disruption, is provided.

\section{System Description}

We consider a system comprised of an arbitrary number $n$ of rigid objects that may be connected one with any of the others and/or with the enviroment through contacts or rotoidal or prismatic joints. Bodies are numbered from 1 to $n$, while the enviroment is assigned the index 0 . Let the location in base frame of the characteristic point of the connection between the $i$-th and $j$-th objects be $c_{i, j} \in \mathbb{R}^{3}$. For prismatic or rotoidal joints, the characteristic point can be chosen as the joint origin in usual Denavit-Hartenberg conventions, while the contact centroid definition of [Bicchi et al., 1993] is used for contacts.

The actuation system is comprised of $q$ motors and $r$ tendons. Tendons always have one end fixed to one of the objects, while the other end may be connected to a motor or to another object $(r \geq q)$. Tendons may be routed through idle pulleys or sheaths, possibly fixed to some of the bodies. The point on body $i$ where tendon $j$ is fixed or is passed through is $\mathbf{v}_{i, j}$. According to the indexing of bodies, the position of the $j$-th tendon actuator is denoted by $v_{0, j}$. Tendons are supposed uniformly stressed (frictionless transmission), and tensions applied to each tendon are collected in a vector $\mathbf{r}=\left(r_{1}, r_{2}, \ldots, r_{r}\right)^{T} \in \mathbb{R}^{r}$. We introduce the shorthand notation $r=\left[r_{i}\right]_{1}^{r}$ for similar juxtaposed vectors and matrices to be encountered.

Denote with $\tilde{\mathbf{t}}_{i, j} \in \mathbb{R}^{6}$ the force/torque (wrench) exerted on the $i$-th object by the $j$-th object. Further, let $\tilde{t}_{i} \in \mathbb{R}^{6 n}$ contain all vectors $\tilde{t}_{i, j}$ with $j \neq i, \tilde{t}_{i}=$ $\left[\tilde{\mathbf{t}}_{i, j}\right]_{1, j \neq i}^{n}$, and let the external wrench applied on the $i$-th object be $w_{i} \in \mathbb{R}^{6}$ (see fig.1). The overall system balance equation can be written as

$$
\begin{gathered}
\mathbf{w}=-\tilde{\mathbf{G}} \tilde{\mathbf{t}}-\mathbf{T} \mathbf{r} \\
\text { where } \mathbf{w}=\left[\mathbf{w}_{i}\right]_{1}^{n} \in \mathbb{R}^{6 n}, \tilde{\mathbf{t}}=\left[\tilde{\mathbf{t}}_{i}\right]_{1}^{n} \in \mathbb{R}^{6 n^{2}} \text {, and } \\
\tilde{\mathbf{G}}=\operatorname{diag}\left[\tilde{\mathbf{G}}_{i}\right]_{1}^{n} \in \mathbb{R}^{6 n \times 6 n^{2}} \\
\tilde{\mathbf{G}}_{i}=\left[\tilde{\mathbf{G}}_{i, j}^{T}\right]_{1, j \neq i}^{T} \in \mathbb{R}^{6 \times 6 n} \\
\tilde{\mathbf{G}}_{i, j}=\left(\begin{array}{cc}
\mathbf{I}_{3} & 0 \\
c_{i, j} \times \mathbf{I}_{3}
\end{array}\right) \in \mathbb{R}^{6 \times 6} \\
\mathbf{T} \in \mathbb{R}^{6 n \times r} .
\end{gathered}
$$

The construction of matrix $T$ is detailed in the appendix. To take into account that not all tendons are directly actuated, a suitable selection matrix $\Gamma \in \mathbb{R}^{q \times r}$ is introduced with $\Gamma_{i, j}=1$ if the $j$-th tendon is directly connected to the $i$-th motor, and $\Gamma_{i, j}=0$ otherwise. Letting $\tau$ denote the vector of tractions of the $q$ motors, we write

$$
\tau=\Gamma \mathbf{r} .
$$

To model costraints due to joints and to contacts between bodies, consider two reference frames $C_{i, j}$ and $C_{j, i}$ fixed with the object $i$ and the object $j$, respectively, and centered in $c_{i, j}$. Corresponding to a small displacement $\Delta \mathrm{l}_{i}$ and rotation $\Delta \Phi_{i}$ of the $i$-th object (summarized in the twist vector $\Delta \mathbf{u}_{i}=$ $\left.\left(\Delta \mathrm{l}_{i}^{T}, \Delta \Phi_{i}^{T}\right)^{T} \in \mathbb{R}^{6}\right)$, frame $C_{i, j}$ is displaced by $\Delta \mathbf{v}_{i, j}$ and rotated by $\Delta \phi_{i, j}$

$$
\Delta \mathbf{x}_{i, j}=\left(\begin{array}{c}
\Delta \mathbf{v}_{i, j} \\
\Delta \phi_{i, j}
\end{array}\right)=\tilde{\mathbf{G}}_{i, j}^{T} \Delta \mathbf{u}_{i}
$$

Connection (joint or contact) constraints impose that some components of the relative displacement $\Delta x_{i, j}-$ $\Delta x_{j, i}$ are opposed by reaction forces while others are left free, depending upon the type of connection. The structure of the connection between the $i$-th and $j-$ th bodies is summarized by another selection matrix, $\mathbf{H}_{i, j} \in \mathbb{R}^{t_{i j} \times 6}$ such that forces and torques mutually exerted at the connection are written as

$$
\mathbf{t}_{i, j}=\mathbf{K}_{i, j} \mathbf{H}_{i, j}\left(\Delta \mathbf{x}_{i, j}-\Delta \mathbf{x}_{j, i}\right)+\hat{t}_{i, j}
$$

where the stiffness matrix $\mathbf{K}_{i, j} \in \mathbb{R}^{t_{i, j} \times t_{i, j}}$ incorporates the structural elasticity of the connection elements ("virtual springs"), and $\hat{t}_{i, j}$ is the contact force when the relative displacement is zero. Note that in eq. (2), $t_{i, j} \in \mathbb{R}^{t_{i j}}$ differs from $\tilde{t}_{i, j}=\mathbf{H}_{i, j}^{T} \mathbf{t}_{i, j} \in \mathbb{R}^{6}$ in that only the components of connection wrench relevant to the interaction are present. The $n^{2}$ equation (2) can be summarized, using eq.(1), as

$$
\mathbf{t}=\mathbf{K H X} \Delta \mathbf{x}+\hat{\mathbf{t}}=\mathbf{K H X} \tilde{\mathbf{G}}^{T} \Delta \mathbf{u}+\hat{\mathbf{t}}
$$

where $\Delta x=\left[\Delta x_{i}\right]_{1}^{n}$ and $\Delta x_{i}=\left[\Delta x_{i, j}\right]_{1, j \neq i}^{n} \cdot$ Matrix $\mathbf{X} \in \mathbb{R}^{6 n^{2} \times 6 n^{2}}$ selects appropriate combinations 
of displacements $\Delta x_{i, j}$. The structure of $\mathbf{H} \in \mathbb{R}^{* \times 6 n^{2}}$, $K \in \mathbb{R}^{d x}$, and $X$ is described in the appendix.

The model of tendon elasticity is obtained introducing the tendon relative displacement $\Delta x_{\text {, and the }}$ displacements imposed by motors on tendon ends, $\Delta \mathbf{q}$. Accordingly, the tension of tendons $\mathbf{r}$ is

$$
\mathbf{r}=\mathbf{K}_{\mathbf{r}} \Delta \mathbf{x}_{\mathbf{p}}+\hat{\mathbf{r}}=\mathbf{K}_{\mathbf{r}}\left(\mathbf{T}^{T} \Delta \mathbf{u}-\Gamma^{T} \Delta \mathbf{q}\right)+\hat{\mathbf{r}} .
$$

where $\hat{\mathbf{r}}$ are the tensions in the reference configuration $\Delta x_{p}=0$. The diagonal stiffness matrix $\mathbf{K}_{r} \in \mathbb{R}^{r \times p}$ depends on the elastic characteristics of tendons.

In view of the above definitions, the model of the system to be studied can be summarized by the following equations:

$$
\begin{aligned}
\mathbf{w} & =-\tilde{\mathbf{G}} \overline{\mathbf{t}}-\mathbf{T r}=-\left(\tilde{\mathbf{G}} \mathbf{H}^{T} \mathbf{T}\right)\left(\begin{array}{l}
\mathbf{t} \\
\mathbf{r}
\end{array}\right) \stackrel{\text { dof }}{=}-\overline{\mathbf{G}} \overline{\mathbf{t}} \\
\boldsymbol{\tau} & =\Gamma \mathbf{r} \\
\mathbf{t} & =\mathbf{K} \mathbf{H} \mathbf{X} \Delta \mathbf{x}+\hat{\mathbf{t}}=\mathbf{K} \mathbf{H} \times \tilde{\mathbf{G}}^{T} \Delta \mathbf{u}+\hat{\mathbf{t}} \\
\mathbf{r} & =\mathbf{K}_{\boldsymbol{r}} \Delta \mathbf{x}_{\boldsymbol{q}}+\hat{\mathbf{r}}=\mathbf{K}_{\boldsymbol{r}}\left(\mathbf{T}^{T} \Delta \mathbf{u}-\Gamma^{T} \Delta \mathbf{q}\right)+\hat{\mathbf{r}}
\end{aligned}
$$

\section{Force Distribution}

The force distribution problem consists of describing the general solution to eq.(3), a linear system of $6 n$ equation in $+r$ unknowns. Note that eq.(3) admits solution only if $w$ belongs to the range space of $\overline{\mathbf{G}}$. Whithout loss of generality, we assume that $\mathcal{R}(\overline{\mathbf{G}})=\mathbb{R}^{\text {on }}$. The general solution of eq.(3) can be written as the sum of a particular solution and an homogeneous solution.

\subsection{Particular solution}

The particular solution is in general not unique, since $\overline{\mathbf{G}}$ admits infinitely many right inverses. However, we expect a unique solution to the following problem:

Assume the system is in an equilibrium configuration, under a set of external loads $\hat{w}$, with contact forces $\hat{\mathbf{t}}$ and tensions of tendons $\hat{\mathbf{x}}$. Determine the contact forces and tendon tensions at the equilibrium that the system reaches when an additional load $\mathbf{w}$ is applied, while the actuator position $\mathrm{q}$ is kept constant. follows

$$
\begin{aligned}
& \overline{\mathbf{t}}=\left(\begin{array}{l}
\mathbf{t} \\
\mathbf{r}
\end{array}\right)=\left(\begin{array}{cc}
\mathbf{K} & 0 \\
0 & \mathbf{K}_{\mathbf{r}}
\end{array}\right)\left(\begin{array}{l}
\mathbf{G}^{T} \\
\mathbf{T}^{T}
\end{array}\right) \Delta \mathbf{u}+\left(\begin{array}{c}
\hat{\mathbf{t}} \\
\hat{\mathbf{r}}
\end{array}\right) \stackrel{\text { def }}{=} \\
& \stackrel{\text { def }}{=} \overline{\mathbf{K}} \overline{\mathbf{G}}_{z}^{T} \Delta \mathbf{u}+\hat{\overline{\mathbf{t}}},
\end{aligned}
$$

where $G_{\star}=H X \tilde{G}^{T}$. Substituting eq.(7) in eq.(3), we have

$$
\mathbf{w}+\hat{\mathbf{w}}=-\overline{\mathbf{G}} \overline{\mathbf{K}} \overline{\mathbf{G}}_{\mathbf{z}}^{\mathbf{T}} \Delta \mathbf{u}-\overline{\mathbf{G}} \hat{\mathbf{t}} .
$$

Hence, recalling that $\overline{\mathbf{G}}$ is assumed full row rank and $\overline{\mathbf{K}}$ is invertible,

$$
\overline{\mathbf{t}}=-\overline{\mathbf{K}} \overline{\mathbf{G}}_{\mathbf{w}}^{\mathbf{T}}\left(\overline{\mathbf{G}} \overline{\mathbf{K}} \overline{\mathbf{G}}_{z}^{T}\right)^{-1} \mathbf{w}+\hat{\overline{\mathbf{t}}} \stackrel{\text { def }}{=}-\overline{\mathbf{G}}_{\mathbf{K}}^{R} \mathbf{w}+\hat{\mathbf{t}}
$$

where $\overline{\mathbf{G}}_{\mathbf{K}}^{R}$ is the $\overline{\mathbf{K}}$-weighted right inverse of $\overline{\mathbf{G}}$. The tendon tensions and contact forces between any pair of bodies caused by the external wrench $w$ can be easily recovered from the definition of $\bar{t}$.

\subsection{Homogeneous solution}

Homogeneous solutions of eq.(3) correspond to tendon tensions and connection forces that counterbalance each other, thus not affecting the overall equilibrinm of the system. However, internal forces are of fundamental concern in grasp planning, since disruption of rolling-pair joints or slippage and loss of grasp stability can often be avoided only through effective management of internal forces. In analogy with systems of whole-limb manipulators without tendons discussed in [Bicchi, 1993], in the present case we may be confronted with the impossibility of arbitrarily controlling every combination of internal forces in the nullspace of $\mathbf{G}$. Among internal tensions-forces, co-contraction tendon tensions can be further distinguished as those that do not affect contact forces between the links and the manipulated objects. Co-contraction tensions must be used to keep tensions positive in each tendon (to avoid them to go slack), and to keep contact between different links in the limbs (when e.g. a rollingpair joint is used).

\subsubsection{Active internal tensions and forces}

Let us rewrite eq.(5) and (6) as

$$
\begin{aligned}
& \overline{\mathbf{t}}=\left(\begin{array}{l}
\mathbf{t} \\
\mathbf{r}
\end{array}\right)=\left(\begin{array}{cc}
\mathbf{K G}_{\mathbf{q}} & \mathbf{0} \\
\mathbf{K}_{\boldsymbol{r}} \mathbf{T}^{T} & -\mathbf{K}_{\boldsymbol{r}} \Gamma^{T}
\end{array}\right)\left(\begin{array}{c}
\Delta \mathbf{u} \\
\Delta \mathbf{q}
\end{array}\right) \stackrel{\text { def }}{=} \\
& \stackrel{\text { def }}{=} \mathbf{M}\left(\begin{array}{c}
\Delta \mathbf{u} \\
\Delta \mathbf{q}
\end{array}\right)
\end{aligned}
$$

We now prove that every active internal (contact and tendon) force can be written as the product of a basis matrix $\mathbf{E}$ times an arbitrary coefficient vector $y$ of suitable dimension. In fact, consider an equilibrium configuration of the system under the wrench $\hat{w}$ and let $\hat{t}, \hat{q}$ be the connection/tendon forces and positions of tendon actuated ends, respectively. Let $\delta u$ be a vector containing virtual displacements of the objects compatible with all connection costraints. Applying the Principle of Virtual Work and eq.(3), we have

$$
\hat{\mathbf{w}}^{\boldsymbol{T}} \delta \mathbf{u}=\hat{\overrightarrow{\mathbf{t}}}^{T} \overline{\mathbf{G}}^{\mathrm{T}} \delta \mathbf{u}=0, \forall \delta \mathbf{u}
$$

Perturbe the equilibrium configuration by imposing displacements of the actuated ends of tendons by $\Delta q$ and let $\Delta \bar{t}, \Delta \mathbf{u}$ be the change of tensions and forces and the change of position of bodies, respectively. A new equilibrium configuration, under the same set of external forces $\hat{w}$, will be reached on condition that the P.V.W. is satisfied:

$$
\hat{\mathbf{w}}^{T} \delta \mathrm{u}=\left(\hat{\overline{\mathbf{t}}}^{T}+\Delta \overline{\mathrm{t}}^{T}\right) \overline{\mathrm{G}}^{T} \delta \mathrm{u}=\Delta \overline{\mathbf{t}}^{T} \overline{\mathbf{G}}^{T} \delta \mathrm{u}=0, \forall \delta \mathrm{u}
$$

Substituting eq.(8), the P.V.W. condition is rewritten as

$$
\overline{\mathbf{G}} \mathbf{M}\left(\begin{array}{c}
\Delta \mathbf{u} \\
\Delta \mathbf{q}
\end{array}\right)=\mathbf{0}
$$


Let $\mathbf{B} \in \mathbb{R}^{(6 n+q) \times b}$ be a matrix whose columns span the nullspace of $\overline{\mathbf{G}} \mathbf{M}$; the subspace of internal forces that can be obtained at stesdy-state after a displacement of the tendon ends is commanded, i.e. the subspace of active internal forces $\mathcal{F}_{h a}$, is given by the range space of $\mathrm{MB}$. A basis matrix $\mathbf{E} \in \mathbb{R}^{\text {exe }}$ is obtained by using only the independent columns of the product $\mathbf{M B}$, and can be partitioned as $\mathbf{E}=$ $\left(\left[\mathbf{E}_{i}\right]_{1}^{n} \mathbf{E}_{r}\right)$, with $\mathbf{E}_{i}=\left[\mathbf{E}_{i, j}\right]_{1, j \neq i}^{n}$. Therefore,

$$
\mathcal{F}_{\text {ha }}=\left\{\overline{\mathbf{t}}: \mathbf{E} \mathbf{y}, \mathbf{y} \in \mathbb{R}^{\mathbf{e}}\right\}
$$

Blocks $\mathbf{E}_{i, j} \in \mathbb{R}^{t_{i, j} \times \boldsymbol{e}}$ correspond to contact forces between the $i$-th and the $j$-th body contributing to active internal forces, while block $\mathrm{E}_{\tau}$ contains the corresponding tensions of tendons necessary to apply the internal forces. Note that the set of the "co-contraction" forces $\mathcal{F}_{c e}$ is the subspace of active internal forces that do not change the forces between links and the manipulated object:

$$
\begin{aligned}
\mathcal{F}_{c e}=\left\{\overline{\mathbf{t}}: \overline{\mathbf{t}}=\mathbf{E} \mathbf{y}, \mathbf{y} \in \mathbb{R}^{e}, \mathbf{E}_{i, j} \mathbf{y}=0\right. \\
\\
\text { if body } i \text { or } j \text { is "object" }\}
\end{aligned}
$$

\subsubsection{Preload internal forces}

We consider preload tensions and forces as those internal tensions and forces that can not be actively controlled by means of motor displacements. Preloading a system can avoid slippage due to a low value of non controllable normal forces. In the analysis of systems with tendinous structure and rolling pairs, preloading can be used to model the effects of articular ligaments. Letting $\Gamma_{T}=(0 \Gamma) \in \mathbb{R}^{g \times(\bullet+r)}$, eq. (4) is rewritten as $\tau=\Gamma_{T} \bar{t}$ and eq. (5) e (6) can be assembled in

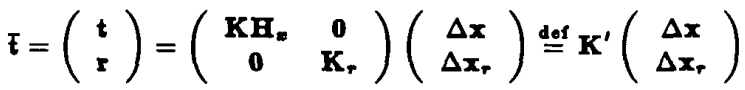

Because every preload force is internal and not controllable by motors, the following relations must be verified

$$
\begin{aligned}
\overline{\mathbf{G}} \mathbf{K}^{\prime}\left(\begin{array}{c}
\Delta \mathbf{x} \\
\Delta \mathbf{x}_{\mathbf{r}}
\end{array}\right) & =\mathbf{0} ; \\
\Gamma_{\boldsymbol{T}} \mathbf{K}^{\prime}\left(\begin{array}{c}
\Delta \mathbf{x} \\
\Delta \mathbf{x}_{\mathbf{r}}
\end{array}\right) & =\mathbf{0} ;
\end{aligned}
$$

The subspace of preload tensions and forces $\mathcal{F}_{h p}$ is therefore given by

$$
\mathcal{F}_{h p}=\left\{\overline{\mathbf{t}}: \overline{\mathbf{t}}=\mathbf{K}^{\prime} \mathbf{y}, \mathbf{y} \in \mathcal{N}\left(\overline{\mathbf{G}} \mathbf{K}^{\prime}\right) \cap \mathcal{N}\left(\Gamma_{T} \mathbf{K}^{\prime}\right)\right\}
$$

where $\mathcal{N}(\cdot)$ indicates the nullspace of the argument. To evaluate a basis matrix $\mathbf{P}$ of the preload subspace, an algorithm for the such as that described in [Bicchi 1993] should be applied. Details are skipped for space limitations.

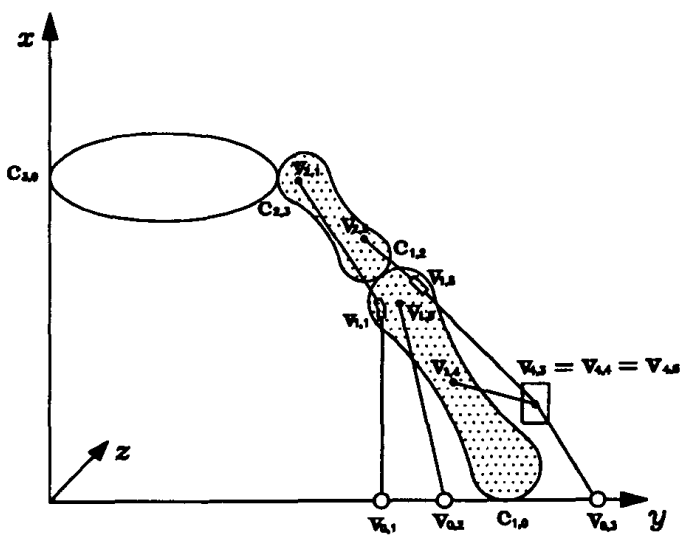

Figure 2: A finger with two phalanges and three tendons manipulating an object against a wall.

\section{Example}

Consider the system of fig. 2 comprised of a finger with two phalanges pushing an object against a wall. The actuation system is comprised of three tendons. To deal with the bifurcation of one tendon, one fictitious link and two fictitious tendons are introduced. The coordinates of contact centroids are (in conventional measure units)

$$
\begin{gathered}
c_{1,0}=\left(\begin{array}{lll}
0 & 20 & 0
\end{array}\right)^{T} ; c_{1,2}=c_{2,1}=\left(\begin{array}{lll}
10.4 & 14 & 0
\end{array}\right)^{T} ; \\
c_{2,3}=c_{8,2}=\left(\begin{array}{llll}
14.6 & 9.76 & 0
\end{array}\right)^{T} ; c_{3,0}=\left(\begin{array}{lll}
14.6 & 0 & 0
\end{array}\right)^{T}
\end{gathered}
$$

Rolling-pair "hinges" in $c_{1,0}$ and $c_{1,2}$ allow only rotations around the $z$ axis, hence $n_{1,0}=n_{1,2}=\left(\begin{array}{lll}0 & 0 & 1\end{array}\right)^{T}$. Contacts $c_{2,3}$ e $c_{3,0}$ are of "soft finger" type with normal directions $\mathbf{n}_{2,3}=\left(\begin{array}{lll}0 & 1 & 0\end{array}\right)^{T} ; \mathbf{n}_{3,0}=\left(\begin{array}{lll}0 & 1 & 0\end{array}\right)^{T}$. The tendon arrangement is described as follows:

Tendon 1 is connected to finger 2 at $v_{2,1}=$ $(14.59 .90)^{T}$, goes through a conduit fixed to finger 1 at $\mathbf{v}_{1,1}=(9.412 .30)^{T}$, and is connected to motor 1 at $\mathbf{v}_{0,1}=\left(\begin{array}{lll}0 & 12.3 & 0\end{array}\right)^{T}$;

Tendon 2 is connected to finger 1 and to motor 2 at

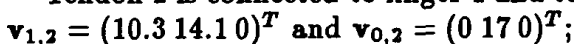

Tendon 3 is connected to object 4 (fictitious) at $v_{4,3}=(3.220 .50)^{T}$ and to motor 3 at $v_{0,3}=(023.70)^{T}$;

Tendon 4 is connected to object 4 (fictitious) at $v_{4,4}=(3.220 .50)^{T}$ and to finger 1 at $v_{0,3}=(5.2170)^{T}$; Tendon 5 is connected to finger 2 at $\mathbf{v}_{2,5}=$ $(10.513 .90)^{T}$, goes through a sheath fixed to finger 1 at $v_{1,5}=(11.415 .70)^{T}$, and is connected to object 4 (fictitious) at $\mathrm{v}_{4,5}=(3.2120 .50)^{T}$.

Dimensions of vectors and matrices are as follows: $\mathbf{t}=[\mathbf{t}]_{1}^{4} \in \mathbb{R}^{3} 7 ; \mathbf{G} \in \mathbb{R}^{24 \times 37} ; \mathbf{H} \in \mathbb{R}^{37 \times 96} ; \mathbf{K} \in$ $\mathbb{R}^{37 \times 37} ; \mathbf{X} \in \mathbb{R}^{96 \times 86} ; \mathbf{T} \in \mathbb{R}^{24 \times 5} ; \Gamma \in \mathbb{R}^{3 \times 5}$. To clarify notation, the composition of vectors $t_{i}=\left[t_{i, j}\right]_{1, j \neq i}^{4}$ 
is illustrated. The first three elements of $t_{1,0} \in \mathbb{R}^{5}$ contain the $x, y$, and $z$ components of the force exerted by the enviroment on link 1 , whereas the latter two are the torque components along $x$ and $y$. Note that no torque is applied along the $z$ axis in a hinge joint. Similarly, $t_{1,2} \in \mathbb{R}^{5}$ contains three force components and two torque components exerted by link 2 on link 1. Vectors $t_{1,8}=t_{1,4}=0 \in \mathbb{R}^{1}$ because link 1 is not in contact with objects 3 and link 4. Analogously, $t_{2,0}=t_{2,4}=0 \in \mathbb{R}^{1}$. Vector $t_{2,1} \in \mathbb{R}^{5}$ is opposite to $t_{1,2}$ (force exerted on link 2 by link 1 is opposite to the force exerted on link 1 by 2 ), and $t_{2,8} \in \mathbb{R}^{4}$ contains the $x, y$, and $z$ components of the force exerted by object 3 on link 2 and the torque along $n_{2,3}$. Vectors $t_{3,1}=t_{3,4}=0 \in \mathbb{R}^{1}$ because of non-interaction, $t_{3,2}=-t_{2,3}$, and $t_{3,0} \in \mathbb{R}^{4}$ contains the $x, y$, and $z$ components of the force and the torque along $n_{2,3}$ exerted by the wall on object 3 . Vector $t_{4} \in \mathbb{R}^{4}$ is sero because link 4 is not in contact with any other body.

We assume a value of $100 \mathrm{~N} / \mathrm{cm}$ for stiftness of linear springs, $20 \mathrm{~N} / \mathrm{cm}$ for the stiffness of rotoidal springs, and $10 \mathrm{~N} / \mathrm{cm}$ for stiffness of tendons. Applying the algorithm shown in 3.2.1, the basis of active internal forces is found as:

$$
\begin{gathered}
\mathbf{E}_{1,0}=\left(\begin{array}{cc}
1.9 & 0.76 \\
-1.2 & -0.45 \\
0 & 0 \\
0 & 0 \\
0 & 0
\end{array}\right) \\
\mathbf{E}_{1,2}=-\mathbf{E}_{2,1}=\left(\begin{array}{cc}
-0.29 & 0.05 \\
0.82 & 0.13 \\
0 & 0 \\
0 & 0 \\
0 & 0
\end{array}\right) \\
\mathbf{E}_{2,3}=-\mathbf{E}_{3,2}=\mathbf{E}_{8,0}=\left(\begin{array}{cc}
0 & 0 \\
0.23 & 0 \\
0 & 0 \\
0 & 0
\end{array}\right) \\
\left(\begin{array}{cc}
0.52 & 0.01 \\
0.63 & 0.63 \\
0.79 & 0.26 \\
0.40 & 0.13 \\
0.40 & 0.13
\end{array}\right) \mathbf{E}_{1,3}=\mathbf{E}_{3,1}=\mathbf{E}_{1,4}=\mathbf{E}_{4,1}=0 \\
\mathbf{E}_{2,0}=\mathbf{E}_{2,4}=\mathbf{E}_{4,2}=\mathbf{E}_{8,4}=\mathbf{E}_{4,3}=0 \\
\mathbf{E}_{4,0}=\mathbf{E}_{4,1}=\mathbf{E}_{4,2}=\mathbf{E}_{4,3}=0
\end{gathered}
$$

Note that the second column of $\mathbf{E}$ forms a basis of the active internal co-contraction forces because no forces are applied to object 3 (manipulated object). The preload force basis matrix $\mathbf{P}$ for this example results

$$
\begin{gathered}
\mathbf{P}_{1,0}=\mathbf{P}_{1,2}=-\mathbf{P}_{2,1}=\left(\begin{array}{lllll}
0 & 0 & 0 & 0 & -1
\end{array}\right)^{T} \\
\mathbf{P}_{2,3}=-\mathbf{P}_{8,2}=-\mathbf{P}_{8,0}=\left(\begin{array}{llll}
0 & 0 & 0 & 1
\end{array}\right)^{T} \\
\mathbf{P}_{1,3}=\mathbf{P}_{1,4}=\mathbf{P}_{2,0}=\mathbf{P}_{2,4}=\mathbf{P}_{8,1}=\mathbf{P}_{3,4}=0 \\
\mathbf{P}_{4,0}=\mathbf{P}_{4,1}=\mathbf{P}_{4,2}=\mathbf{P}_{4,3}=0 \quad \mathbf{P}_{r}=\mathbf{0}_{8 \times 1}
\end{gathered}
$$

Allowed preload forces correspond to torsion of the object between two soft-finger contacts, and is of no concern for the tendinous actuation system.

We finally report the results of an optimiration algorithm (not described here) that is used to calculate the combination of internal forces to minimise the danger of slippage at the contacts and negative tensions in the tendons, when an external unit force is applied to the centre of object 3 in the direction of the $x$ axis. The following values of contact force/torque have been obtained:

$$
\begin{gathered}
t_{1,0}=(22.8-14.5000)^{T} ; t_{1,2}=-t_{2,1}=(-4.411000)^{T} \\
t_{3,0}=(-0.593 .0700)^{T} ; t_{2,3}=-t_{8,2}=(0.413 .0700)^{T} \\
t_{1,8}=t_{8,1}=t_{1,4}=t_{4,1}=t_{2,0}=t_{2,4}=t_{4,2}=0 \\
t_{3,4}=t_{4,3}=t_{4,0}=t_{4,1}=t_{4,2}=t_{4,3}=0
\end{gathered}
$$

$r_{1}=7.8, r_{2}=4.26, r_{3}=10.02, r_{4}=5.18, r_{5}=$ 5.18. This results show that all tendons are correctly streched, that forces at the hinges are compressive (so that a rolling-pair joint can be adopted in the design), and that slippage of the object relative to the finger and to the wall is prevented.

\section{Conclusions}

The method presented is very general and allows to attack in a unified manner a very broad variety of mechanism. Although only discussed in a quasi-static setting, it can be easily modified to model the dynamics of such systems ([Wittemburg, 1977]). One of the main drawbacks of the method is the introduction of rather large matrices even for simple systems, as shown in the example above. This is a price we pay to generality, and more computationally-efficient formulations can be derived by specializing the treatment of particular cases.

\section{Acknowledgments}

This work was supported by Progetto Finalizsato Robotica CNR, Italy, Contracts No. 92.01079.PF67 and 92.01047.PF67 (University of Pisa) .

\section{Appendix}

Matrix T. Matrix $\mathbf{T} \in \mathbb{R}^{\mathbf{0 n \times r}}$ can be partitioned in $6 \times 1$-blocks $\mathbf{T}_{i, j}, i=1, \ldots, n, j=1, \ldots, r$, that can be evaluated by the following rule:

- ) if the tendon $j$ is not connected to the $i$-th link and does not pass through a sheath fixed to the $i$-th link, $\mathbf{T}_{i, j}=\mathbf{0}_{\mathrm{\theta} \times 1}$

- ) if the tendon $j$ is connected to the $i$-th link and successively passes through a sheath fixed to the $k$-th link,

$$
\mathbf{T}_{i, j}=\frac{1}{\left\|\mathbf{v}_{k, j}-\mathbf{v}_{i, j}\right\|}\left(\begin{array}{c}
\mathbf{v}_{k, j}-\mathbf{v}_{i, j} \\
\mathbf{v}_{i, j} \times \mathbf{v}_{k, j}
\end{array}\right)
$$

- ) if the tendon $j$ is connected to (or passes through) link $h$, passes through link $i$ and is connected to (or passes through) link $k$,

$$
\mathbf{T}_{i, j}=\frac{1}{\left\|\mathbf{v}_{h, j}+\mathbf{v}_{h, j}-2 \mathbf{v}_{i, j}\right\|}\left(\begin{array}{c}
\mathbf{v}_{h, j}+\mathbf{v}_{h, j}-2 \mathbf{v}_{i, j} \\
\mathbf{v}_{i, j} \times\left(\mathbf{v}_{h, j}+\mathbf{v}_{k, j}\right)
\end{array}\right)
$$


Matrix $\mathbf{H}$ Matrix $\mathbf{H} \in \mathbb{R}^{2 \times 6 n^{2}}$ is nested blockdiagonal, with diagonal blocks $\mathbf{H}_{i} \in \mathbb{R}^{j i \times 6 n}$ defined as $\mathbf{H}_{i}=\operatorname{diag}\left[\mathbf{H}_{i, j}\right]_{i, j \neq i}^{n}$. Blocks $\mathbf{H}_{i, j} \in \mathbb{R}^{t_{i, j} \times \mathbf{6}} \mathrm{em}-$ body the motion constraints imposed by the particular type of connection between bodies $i$ and $j$. Common contact types are

$$
\begin{array}{ll}
\mathbf{H}_{i, j}=\mathbf{I}_{3} & \text { for "hard finger" } \\
\mathbf{H}_{i, j}=\mathbf{I}_{8} & \text { for "complete cost } \\
\mathbf{H}_{i, j}=\left(\begin{array}{l|l}
\mathbf{I}_{3} & \mathbf{0} \\
\hline \mathbf{0} & \mathbf{n}_{i, j}
\end{array}\right) & \text { for "soft finger"; }
\end{array}
$$

where $\mathbf{n}_{i, j}$ is the direction of the normal between the objects $i$ and $j$. Another important type of connection is the ${ }^{n}$ hingen joint that only allows relative rotation around an axis $n_{i, j}$ :

$$
\mathbf{H}_{i, j}=\left(\begin{array}{c|c}
\mathbf{I}_{3} & \mathbf{0} \\
\hline \mathbf{0} & \mathbf{a}_{i, j} \\
\mathbf{b}_{i, j}
\end{array}\right)
$$

where $a_{i, j}$ e $b_{i, j}$ form a basis of the plane perpendicular to the direction $\mathbf{n}_{i, j}$.

Matrix $K$. The stiffness matrix $K \in \mathbb{R}^{\text {ex }}$ has the same block structure as $\mathbf{H}$. Blocks $\mathbf{K}_{i, j} \in \mathbb{R}^{t_{i, j} \times t_{i, j}}$ are diagonal, and depend upon the type of contact and the stiffness of the virtual spring interposed between objects $i$ and $j$. In the easiest cases the elements of the matrix $K_{i, j}$ are equal to $\mu_{t}$ for linear springs and to $\mu_{r}$ for rotoidal springs. Some examples follow:

$$
\begin{array}{ll}
\mathbf{K}_{i, j}=\mu_{t} \mathbf{I}_{3} & \text { for "hard finger"; } \\
\mathbf{K}_{i, j}=\left(\begin{array}{c|c}
\mu_{t} \mathbf{I}_{3} & 0 \\
\hline 0 & \mu_{r} \mathbf{I}_{3}
\end{array}\right) & \text { for "complete costraint"; } \\
\mathbf{K}_{i, j}=\left(\begin{array}{c|c}
\mu_{t} \mathbf{I}_{3} & 0 \\
\hline 0 & \mu_{r}
\end{array}\right) & \text { for "soft finger"; } \\
\mathbf{K}_{i, j}=\left(\begin{array}{c|c}
\mu_{t} \mathbf{I}_{3} & 0 \\
\hline 0 & \mu_{r} \mathbf{I}_{2}
\end{array}\right) & \text { for "hinge" joints; }
\end{array}
$$

$\mathbf{K}_{\boldsymbol{r}} \in \mathbb{R}^{r \times \boldsymbol{r}}$, the matrix of tendon's elasticity, is also diagonal.

Matrix X: The selection matrix $X \in \mathbb{R}^{6 n^{2} \times 6 n^{2}}$ can be partitioned as follows:

$$
\mathbf{X}=\left(\begin{array}{ccc}
\mathbf{X}_{1,1} & \ldots & \mathbf{x}_{1, n} \\
\ddot{\mathbf{x}}_{n, 1} & \ldots & \ddot{\mathbf{x}}_{n, n}
\end{array}\right)
$$

where the blocks $\mathbf{X}_{i, j} \in \mathbb{R}^{6 n \times 6 n}$ are defined as:

$$
\mathbf{X}_{i, j}=\left(\begin{array}{ccc}
\mathbf{X}_{i, j}^{1,1} & \ldots & \mathbf{X}_{i, j}^{1, n} \\
\ldots . & \ldots & \ldots \\
\mathbf{X}_{i, j}^{n, 1} & \ldots & \mathbf{X}_{i, j}^{n, n}
\end{array}\right)
$$

If $i=j$ (diagonal blocks), $\mathbf{X}_{i, i}$ is evalutated according to the following rules:

- blocks $\mathbf{X}_{i, i}^{h, k}$ with $h \neq k$ (non diagonal blocks) are void $6 \times 6$ matrices;

- blocks $\mathbf{X}_{i, i}^{h, h}$ (diagonal blocks):
- if $i$-th object is in contact with the enviroment, $\mathbf{X}_{i, i}^{1,1}=\mathbf{I}_{\boldsymbol{B}}$;

-- if $i$-th object is in contact with $j$-th object:

--- if $i>j, \mathbf{x}_{i, i}^{j, j}=\mathbf{I}_{6}$;

--- if $i<j, \mathbf{X}_{i, i}^{(j+1),(j+1)}=\mathbf{I}_{6}$; - blocks which do not hold the above properties are void matrices $6 \times 6$.

If $i \neq j$ (non diagonal block) matrix $\mathbf{X}_{i, j}$ :

- if $i$-th object is not in contact with $j$-th object, $\mathbf{X}_{\mathbf{i}, j}=\mathbf{0}_{\text {en } \times \boldsymbol{\theta}_{n}}$;

- if $i$-th object is in contact with $j$-th object then just one block of $\mathbf{X}_{i, j}$ is not void. In particular we have:

$$
\begin{aligned}
& - \text { if } j>i \mathbf{X}_{i, j}^{j,(i+1)}=-\mathbf{I}_{6} ; \\
& -- \text { if } j<i \mathbf{X}_{i, j}^{(j+1), i}=-\mathbf{I}_{6} .
\end{aligned}
$$

\section{References}

L. Barbieri, M. Bergamasco. "Nets of Tendons and Actuators: an Anthropomorphic Model for the Actuation System of Dextrous Robot Hands". IEEE Int. Conf. on Robotics and Automation, 1991.

J. Backer, N. Thakor, K. Gruber. "A Study of Human Hand Tendon Kinematics with Applications to Robot Hand Design". IEEE Int. Conf. on Robotics and Automation, 1986.

A. Bicchi, J.K.Salisbury, D.L.Brock: "Contact Sensing from Force and Torque Measurements", Int. J. of Robotics Research, Vol.12, no.3, 1993.

A. Bicchi: "Force Distribution in Multiple Whole-limb Manipulation", IEEE Int. Conf. on Robotics and Automation, 1993.

H. Buchner, M. Hines, H. Hemani. "A dynamic model for finger interphalangeal coordination", J. Biomechanics, 21:459-468, 1988.

D. Deno, M. Murray, K. Pister, S. Sastry. "Fingerlike Biomedical Robot". IEEE Int. Conf. Robotics and Automation, pag.566-572, 1992.

S. Jacobsen, H. Ko, E. Iversen, C. Davis. "Antagonistic Control of a Tendon Driven Manipulator". IEEE Int. Conf. Robotics and Automation, pag.1334-1339, 1989.

J. Lee, L. Tsai. "The Structural Synthesis of TendonDriven Manipulators Having a Pseudotriangular Structure Matrix". Int. J. Robotics Research, Vol.10, n.1, 1991.

A. Morecky, Z. Busko, H. Gastrold, K. Javorek: "Synthesis and Control of the Anthropomorphic Two-Handed Manipulator", Proc. of the 10th Int. Symp. Industr. Robots, Milan, 1980.

F. Mussa Ivaldi, P. Morasso, R. Zaccaria. "Kinematics Network". B. Cybernetics, 60:1-16, 1988.

J.K. Salisbury: " Kinematic and Force Analysis of Articulated Hands", Rep. STAN-CS-82-91, Stanford Univ., Stanford, CA, 1982.

J. Wittemburg. "Dynamics of Systems of Rigid Bodies", B.G. Teubner, Stuttgart, 1977. 